\title{
OTRA CIUDADANÍA ES POSIBLE: UNA MIRADA A PARTIR DE LA MICROPOLÍTICA
}

\author{
A DIFFERENT CITIZENSHIP IS POSSIBLE: \\ A LOOK FROM MICROPOLITICS
}

\author{
OUTRA CIDADANIA É POSSÍVEL: \\ UM OLHAR BASEADO NA MICROPOLÍTICA
}

\author{
Dunia Gutiérrez Niño ${ }^{1}$ \\ Yaneth Malagón Mayorga ${ }^{2}$ \\ Eusebio Avendaño Avendaño ${ }^{3}$
}

\section{Resumen}

\begin{abstract}
Formar en ciudadanía es uno de los retos más significativos que asume el maestro de hoy, sobre todo porque su labor está siendo orientada intencionalmente por el sistema educativo hacia la formación de capital humano; se está educando más para la productividad, la competitividad y menos para la ciudadanía. El presente artículo es el resultado de un proceso de investigación de carácter cualitativo que tiene como objetivo fundamental la conceptualización de principios pedagógicos y didácticos que puedan orientar procesos de formación ciudadana desde una perspectiva de la ética del cuidado de sí como práctica de libertad. Se desarrolla a partir de una revisión documental del Programa de Competencias Ciudadanas (PCC) formulado por el Ministerio de Educación Nacional (MEN), un análisis crítico de este discurso para problematizar la relación entre poder-saber en este contexto, y una reflexión sobre nuestras prácticas educativas -investigación-acción-. Se propone, entonces, una formación ciudadana una formación ciudadana orientada hacia la micropolítica, desarrollada a partir de principios didácticos como la afectividad, la participación como ágora, el diálogo intersubjetivo y el contexto como aula de clase.
\end{abstract}

Palabras clave: competencia ciudadana; prácticas de libertad; micropolítica; afectividad; diálogo intersubjetivo; participación

1 Psicopedagoga de la Universidad Tecnológica y Pedagógica de Colombia; especialista en Planeación, Gestión y control del Desarrollo Social de la Universidad de la Salle. Magíster en Desarrollo Educativo y Social de la Universidad Pedagógica Nacional y CINDE. Correo electrónico: duniacg27@hotmail.com.

2 Licenciada en Matemáticas y especialista en Educación Matemática de la Universidad Distrital Francisco José de Caldas. Docente de la Escuela Pedagógica Experimental. Magíster en Desarrollo Educativo y Social de la Universidad Pedagógica Nacional y CINDE. Correo electrónico: janeth.malagon@epe.edu.co.

3 Filósofo de la Universidad Nacional de Colombia; especialista en Pedagogía de la Universidad Pedagógica Nacional, docente de la Universidad Distrital Francisco José de Caldas y de la Universidad Pedagógica Nacional. Magíster en Desarrollo Educativo y Social de la Universidad Pedagógica Nacional y CINDE. Correo electrónico: euseavendano@gmail.com. 


\begin{abstract}
Citizenship education is one of the most significant challenges that teachers face today, particularly because their work is being intentionally guided by the education system towards the shaping of human capital; they are training them more for productivity and competitiveness and less for citizenship. This paper is the result of a qualitative research process whose fundamental objective is the conceptualization of pedagogical and didactic principles that are able to guide the citizen education processes from a perspective of ethics of selfcare as a practice of freedom. It is developed from a documentary review of the Citizen Competencies Program (PCC) created by the Ministry of National Education (MEN), a critical analysis of this discourse to problematize the relationship between power-knowledge in this context, and a reflection on our educational practicesresearch-action- We propose, then, a citizen education oriented towards micropolitics, developed from didactic principles such as affectivity, participation as an agora, intersubjective dialogue, and context as a classroom.

Keywords: civic competence; practices of freedom; micropolitics; affectivity; intersubjective dialogue; participation

\section{Resumo}

A formação em cidadania é um dos desafios mais significativos assumidos hoje pelo professor, especialmente porque seu trabalho está sendo intencionalmente norteado pelo sistema educacional para a formação do capital humano; estamos educando mais para produtividade, a competitividade e menos para a cidadania. Este artigo é o resultado de um processo de pesquisa qualitativa cujo principal objetivo foi a conceituação de princípios pedagógicos e didáticos que podem nortear processos de formação cidadã a partir da perspectiva da ética do cuidado de si mesmo como prática da liberdade. Desenvolvemos a partir de uma revisão documental do Programa de Competências Cidadãs (PCC) planejado pelo Ministério da Educação Nacional (MEN), uma análise crítica desse discurso para problematizar a relação entre poder-conhecimento neste contexto, e uma reflexão sobre nossas práticas educativas - pesquisa-ação. Propomos, então, uma formação cidadã orientada para a micropolítica, desenvolvida a partir de princípios didáticos como a afetividade, a participação como uma ágora, o diálogo intersubjetivo e o contexto de sala de aula.
\end{abstract}

Palavras-chave: competência cidadã; práticas de liberdade; micropolítica; afetividade; diálogo intersubjetivo; participação

Fecha de recepción: 16 de agosto de 2017

Fecha de aprobación: 9 de julio de 2018

Para citar este artículo:

Gutiérrez, D., Malagón, Y., y Avendaño, E. (2017.) Otra ciudadanía es posible: una mirada a partir de la micropolítica. Lúdica Pedagógica, 26, 117-128. 


\section{EL CAMINO QUE RECORRIMOS}

Este artículo es producto de la investigación: Otra ciudadanía es posible. Análisis crítico al Programa de Competencias Ciudadanas propuesto por el Ministerio de Educación Nacional, en el marco de la Maestría de Desarrollo Educativo y Social Cinde-UPN. Una investigación de carácter cualitativo que se fundamenta tanto en nuestra experiencia docente como en los documentos presentados por el MEN sobre las orientaciones para la institucionalización de las competencias ciudadanas, la ruta de ejecución y los estándares básicos de competencias ciudadanas (MEN, 2004); además, se tomaron como referentes los aportes teóricos de Alexander Ruiz Silva y Enrique Chaux Torres (2005), entre otros, quienes han dedicado gran parte de su vida académica a investigar sobre el tema de las competencias ciudadanas y a asesorar al MEN en el proceso de diseño e institucionalización del PCC.

El análisis crítico lo desarrollamos a partir de los planteamientos de Michel Foucault, sobre el nacimiento de la biopolítica (2007), quien a través de su método arqueológico y genealógico nos ofreció un panorama detallado sobre el surgimiento del neoliberalismo y con él las políticas capitalistas. De este autor también tomamos como referencia la entrevista que le hicieran Raúl Fornet Betancourt, Helmut Becker y Alfredo Gómez Muller, el 20 de enero de 1984, recogida en el texto "La ética del cuidado de uno mismo como práctica de la libertad" (1984), a partir del cual desarrollamos las nociones de libertad, poder y subjetividad. Por esta misma línea, tomamos los aportes de Gilles Deleuze y Félix Guattari (2004), con los que visibilizamos el trasfondo del paso de las sociedades disciplinarias a las sociedades de control, e identificamos posibles maneras como los sujetos podemos resistir a esas nuevas formas de dominación, a través de las líneas de fuga y la micropolítica. De igual forma, las construcciones teóricas de Humberto Maturana (1994) y Martha Nussbaum (2010), sobre las nociones de democracia y participación, fueron inspiradoras, en la medida en que nos permitieron perfilar una visión distinta de estos dos conceptos tan importantes para la formación ciudadana.

Otros referentes importantes son las críticas de Guillermo Bustamante (2010) a la noción de las competencias en la educación; Paulo Freire (2004), con su propuesta de educación como práctica de la libertad, e Irene Comins (2009), en su llamado a asumir las tareas de atención y cuidado, no solo para con los otros, sino para con nosotros mismos.

El objetivo principal que orientó esta investigación fue proponer unos principios pedagógicos y didácticos que puedan guiar procesos de formación ciudadana, desde una perspectiva de la ética del cuidado de sí como práctica de libertad; y los objetivos específicos que apoyaron este proceso fueron analizar de manera crítica la política actual de formación en competencias ciudadanas en Colombia, identificar las ausencias o insuficiencias del programa para formar en ciudadanía y visibilizar tres estrategias didácticas exitosas de formación ciudadana desarrolladas en tres contextos distintos.

Las tareas científicas que se desarrollaron para dar cumplimiento a los objetivos planteados incluyeron la revisión documental del PCC, con el propósito de ampliar nuestro conocimiento sobre el tema de las competencias ciudadanas, y orientar nuestro camino en una lectura crítica y propositiva de lo encontrado en dichos documentos institucionales. En un segundo momento, se realiza un análisis crítico del PCC a partir de un proceso de análisis del discurso, con el fin de hacer visibles sus componentes y denunciar los efectos que este discurso provoca en los sujetos. En un tercer momento, acudimos a lo que Carr y Kemmis (1988) han definido como investigación-acción, en cuanto que su objeto se centra en nuestras propias prácticas educativas, con el propósito de reflexionar sobre el modo como las entendemos y sus distintas situaciones, de manera particular, en lo que consideramos la formación de nuevas subjetividades, de nuevas maneras de entendernos como ciudadanos en nuestro aquí y ahora concreto (nosotros mismos, en cuanto docentes, y los estudiantes con los que tenemos que ver en nuestros espacios educativos). Finalmente, desarrollamos un proceso propositivo mediante el cual se definen otros posibles principios pedagógicos y didácticos de formación ciudadana que pueden complementar las acciones propuestas por el MEN.

Todo esto con el ánimo de contribuir a la transformación y mejora de nuestro metro cuadrado educativo $\mathrm{y}$, con ello, a la utopía de querer lograr transformaciones del presente de la realidad social en la que nos encontramos inmersos, para dar forma a un futuro distinto (Carr y Kemmis, 1988). 


\section{UNA MIRADA CRÍTICA AL PCC}

En la investigación se retoma el planteamiento de Michel Foucault (2007) sobre cómo con el surgimiento del neoliberalismo se instaura un nuevo arte de gobernar que se caracteriza por "buscar cómo se puede ajustar el ejercicio global del poder político a los principios de una economía de mercado" (p. 157) mediante una serie de intervenciones que deben actuar "sobre la sociedad misma en su trama y su espesor" (p. 88). Este nuevo arte de gobernar esboza un gobierno de sociedad cuyo principio regulador no son las mercancías, sino la competencia. "Es decir que lo que se procura obtener no es una sociedad sometida al efecto mercancía, sino una sociedad sometida a la dinámica competitiva. No una sociedad de supermercado: una sociedad de empresa" (p. 182). Una sociedad en la que el sujeto pasa de ser un simple productor-consumidor a ser un gestor de su propia vida, a través del desarrollo de diversas, habilidades o competencias. Una sociedad en donde las políticas gubernamentales se piensan, se diseñan y se ejecutan en torno al fortalecimiento del capital humano.

Estas nuevas dinámicas de gobierno convirtieron a la educación en el principal dispositivo para materializar el discurso del capital humano; así, fue clave para la investigación hacer un seguimiento y análisis de la inclusión de la categoría competencia en el campo de la educación colombiana, propuesta por algunos organismos internacionales como el Banco Mundial (BM) y la Organización para la Cooperación y el Desarrollo Económicos (OCDE), con la pretensión de orientar la educación hacia la formación de sujetos aptos para el mundo laboral. De este modo, las competencias ciudadanas son una estrategia política para aumentar las capacidades cognitivas y emocionales de los sujetos y aumentar su productividad para llegar a constituirse como empresarios de sí mismos. Formar en competencias ciudadanas es incidir en las estructuras más íntimas de los individuos de tal forma que se pueda garantizar un adecuado proceso de subjetivación en torno a la dinámica del mercado.

Dentro del análisis crítico se encontraron insuficiencias en dos sentidos: a nivel conceptual y a nivel práctico o de estrategias de materialización del discurso. A nivel conceptual se identifican algunas debilidades en las principales nociones que definen el PCC como ciudadanía, democracia, participación, libertad, auto- nomía y poder, encontrándose en ellas un discurso intangible que va en contravía de los objetivos del PCC como estrategia para la consolidación de una subjetividad para la democracia. En segundo sentido, se desarrolla una crítica a algunas de las estrategias que propone el MEN para materializar el PCC, como la definición de unos estándares básicos de competencias ciudadanas, la réplica del modelo democrático en la escuela y la interiorización de las normas. Estrategias que también contradicen el discurso de la participación, la autonomía y la libertad.

Los hallazgos del recorrido: develando verdades y buscando salidas

Como resultado de este ejercicio crítico, reflexivo y propositivo, hemos construido una serie de planteamientos teóricos y prácticos que pueden servir de base para continuar con esta ardua tarea de repensar las prácticas educativas.

\section{Sobre la noción de competencias en la educación}

A partir del análisis crítico desarrollado sobre los fundamentos del PCC, se logra comprender que este programa es una estrategia gubernamental con la que se busca hacer de la educación un dispositivo para fortalecer el capital humano. A través de las competencias ciudadanas se busca conducir hasta las más íntimas conductas del sujeto; y en esta medida, lograr que pueda configurarse en un empresario de sí mismo y agente activo dentro de la dinámica neoliberal, teniendo en cuenta que el neoliberalismo se propone hacer funcionar la vida entera del hombre alrededor de la dinámica capitalista de producir, consumir, competir y gestionar su propio bienestar y sus propios riesgos.

Las competencias ciudadanas, entendidas como el conjunto de conocimientos, actitudes, habilidades que el sujeto necesita desarrollar para poder ejercer plenamente la ciudadanía, son parte de las inversiones estratégicas que el gobierno hace para potencializar el capital innato de los sujetos; y de esta manera, formar esa idoneidad- máquina que va a producir ingresos. Es más, para la nueva "gubernamentalidad" es prioritario hacer inversiones educativas que provean a los individuos desde niños de una idoneidad que les permita, en su edad adulta, ser sujetos competentes para la sociedad capitalista. 
Si se tiene en cuenta que el discurso de las competencias es una iniciativa promovida por organismos internacionales de carácter económico, como la OCDE y el BM, queda claro que las pretensiones del PCC no están orientadas hacia la formación de nuevas subjetividades para la democracia como lo plantea el discurso del MEN, sino que su propósito se centra en la inclusión de los sujetos en el mundo laboral.

\section{Sobre las insuficiencias de los}

fundamentos teóricos del discurso de

las competencias ciudadanas

El PCC se fundamenta legal y teóricamente en dos discursos: el de la Constitución política de Colombia de 1991 (CPC) y el de los derechos humanos. Los dos, aunque muy promisorios, están cimentados en un concepto de ciudadano ideal, perfecto, libre de cualquier tipo de conflicto y capaz de autodeterminarse; una idea de ciudadano que invisibiliza la complejidad de la vida humana. En esa medida, desarrollar procesos de formación ciudadana a partir de dichos discursos de carácter abstracto, en nada contribuye para que los estudiantes desarrollen una conciencia crítica, reflexiva y propositiva sobre su realidad. Realidad que nada tiene que ver con tal discurso.

Pretender formar en ciudadanía, a partir de un discurso que no representa de ninguna manera la realidad de los contextos en los que habitan los sujetos, es perfilar una enseñanza vacía y poco motivante, que se convierte en un simple proceso de reproducción de contenidos, para cumplir con el requisito de dar cuenta de los aprendizajes a través de las evaluaciones masivas o Pruebas Saber, con las que el MEN mide el nivel de apropiación del discurso hegemónico por parte de los estudiantes. Además, mostrar estos discursos como la única utopía que la humanidad debe alcanzar es también una forma de negar la posibilidad de que los sujetos se interesen por crear otras maneras de existencia.

Sobre las nociones que fundamentan el discurso de las competencias ciudadanas

Los aspectos más relevantes que logramos visibilizar con el desarrollo del análisis crítico del Pcc fueron las insuficiencias con relación a las nociones que fundamentan dicho programa: ciudadanía, democracia, participación, libertad, autonomía y poder. En estas se identifican una serie de contradiccio- nes que desbordan los propósitos y terminan legitimando un ciudadano distinto a lo proyectado; un ciudadano pasivo, individualista, obediente, acrítico y conformista.

Por un lado, se habla al estudiante de libertad, participación, reflexión crítica y propositiva, etc.; y, por el otro, se le condiciona para que actúe bajo las imposiciones de la institución, que a la vez funciona bajo las condiciones del discurso gubernamental.

La ciudadanía definida por el MEN, como la posibilidad que tienen los sujetos de vivir en libertad, compartir un espacio común y asumir un rol más activo en los procesos sociopolíticos, termina siendo, en el mejor de los casos, asumida por los ciudadanos como la obligación de cumplir con los deberes, respetar la autoridad, pagar impuestos, y demás acciones que impliquen obedecer lo establecido por los Gobiernos de turno. No hay en los ciudadanos muestras de una consciencia colectiva, de una identidad ni de un compromiso con su contexto.

A partir de las reflexiones sobre nuestras prácticas pedagógicas, se plantea que la ciudadanía debe darse en un ambiente en el cual el otro surge como legítimo otro en convivencia con uno (Maturana, 1994); de esta manera estaríamos reconociendo a todos los ciudadanos, en su heterogeneidad. Legitimar al otro descarta la idea de discriminar y ratifica que todos somos seres diversos y particulares.

Por otro lado, la democracia y la participación, íntimamente ligadas, son definidas por el PCC como la posibilidad de participar en la construcción del proyecto político de la ciudad o territorio a través de diversos mecanismos como el voto, el referendo, iniciativas populares, revocatoria del mandato, entre otros; pero, dentro de las orientaciones del MEN, se termina resaltando la importancia de la democracia representativa y el ejercicio electoral, al proponer como una de las principales estrategias de formación ciudadana la réplica del modelo que se presenta en el país y que desafortunadamente ha estado permeado por el clientelismo y la corrupción.

Con relación a la noción de libertad definida por el PCC como una condición innata que inviste al ciudadano de la posibilidad de disfrutar de unos derechos, de moverse entre lugares, ideas y gustos y como un valor absoluto que debe ser asumido por todos 
de la misma manera, cabe anotar que es una de las nociones que más distorsiona los procesos de formación ciudadana en varios sentidos. Por un lado, porque los estudiantes, al creerse libres por naturaleza, no logran reconocer y reflexionar sobre las condiciones políticas que determinan su existencia, y terminan adoptando una posición cómoda y conformista que anula cualquier posibilidad de emprender ejercicios de resistencia; y por el otro, porque los obliga a contemplar una sola manera de existencia que les niega la posibilidad de proyectar y materializar otros modos de vivir, alternos a los establecidos por la sociedad hegemónica.

En este sentido, se plantea que los procesos de formación ciudadana, para que sean significativos y permitan la constitución de un sujeto distinto capaz de participar en la construcción de otra ciudadanía, deben partir de la idea de que la libertad no es una condición natural, sino un estado que todo sujeto debe alcanzar a través de unas relaciones de poder bien orientadas hacia el cuidado de sí, lo que implica unas relaciones de dominio sobre sí mismo. Los estudiantes necesitan comprender que la libertad se conquista a partir de ejercicios de resistencia que les permita superar los modos de vida impuestos, y en esa medida establecer sus propios modos de existencia.

Acogiendo los planteamientos de Deleuze y Guattari (2004), la libertad debe ser entendida como aquello que resulta de un devenir minoritario, como un asunto político que recurre a todo un trabajo de potencia, a una micropolítica activa que nos permite transformarnos a nosotros mismos a partir de la autonomía.

Con relación a la autonomía definida por el PCC, como la posibilidad de los sujetos de construir un proyecto de vida propio, es claro que a través de las prácticas escolares que implican en todo momento obediencia, y de los estándares curriculares donde se definen los aprendizajes básicos que los estudiantes deben adquirir, la posibilidad de diseñar un plan de vida propio es uno de los objetivos inalcanzables en la medida en que dichos estándares buscan constituir un tipo de sujeto que responda a los intereses del discurso dominante. El sujeto no tiene opción de diseñar su plan de vida; los Gobiernos de turno ya se lo han diseñado.

Respecto de la noción de poder que se presenta en los distintos documentos del MEN y en la Constitución Política de Colombia como un ejercicio de autoridad o dominio sobre otros, delegado generalmente a los representantes de las diferentes instancias elegidos "democráticamente" — darle poder a una institución o al Estado-, es de anotar que es una concepción de poder que no posibilita a los sujetos una comprensión de su rol como sujetos políticos. Es una comprensión del poder que, en términos de Foucault, se acerca más a estados de dominación en la medida en que muchas veces estas relaciones se encuentran bloqueadas y fijadas por aquellos que ostentan la autoridad y son quienes de manera arbitraria definen lo que los sujetos deben ser y hacer.

Es usual que los discursos de formación ciudadana estén orientados por un lado a explicar las ramas del poder público como instancias que definen los destinos del país, y por el otro, a inculcar el respeto por la autoridad. Estas cuestiones han legitimado en la mente de los estudiantes que el poder es algo externo a ellos, y que es poseído cuando se está en alguna posición económica, social o política distinta a la de las mayorías. En esa medida, se limita su compresión del poder. Así, otra de las nociones que debe ser reorientada dentro del programa de formación ciudadana es la de poder, persuadiendo a los estudiantes para que lo asuman como una situación estratégica, o como la posibilidad que tienen de conducirse y transformarse a sí mismos y a los otros a través de unas relaciones de poder que están presentes en todas las relaciones humanas, tomando diferentes formas. Llevarlos a comprender que este no se posee, sino que se ejerce, para intentar guiar la conducta de los otros de manera positiva.

Se debe hablar, entonces, de relaciones de poder móviles, que permiten a los individuos emprender ejercicios de resistencia con el objetivo de invertir las situaciones que los oprimen; unas relaciones de poder que solo podrán darse en la medida en que los sujetos son libres; relaciones de poder que hay que controlar mediante prácticas de libertad para evitar caer en estados de dominación (Foucault, 1994). 
Sobre las estrategias propuestas para materializar el discurso de las competencias ciudadanas

Las estrategias tomadas para la reflexión son la réplica del modelo de democracia dentro de la escuela, el modelo de internalización de las normas y la definición de unos estándares básicos de competencias ciudadanas.

Sobre la réplica del modelo de democracia en la escuela a través del consejo estudiantil y otros estamentos, planteamos que es una de las estrategias más equívocas, teniendo en cuenta que se privilegia una representatividad que le resta responsabilidades a la mayoría de los actores de la comunidad educativa, ya que se relegan a unos pocos las tareas de participar y decidir. Además, la voz de todos los actores no está en igualdad de condiciones, pues prevalece la de quienes ostentan la autoridad; en este caso, los maestros y directivos. De otro lado, queda en evidencia que lo único que se logra es que los estudiantes, lejos de tomar conciencia de su rol social, repliquen las prácticas proselitistas que se dan en los escenarios "democráticos" reales y terminen siendo apáticos e indiferentes frente a su condición de sujetos políticos.

Respecto de la internalización de las normas, si bien es cierto que las normas son de vital importancia para garantizar el orden social, desarrollar un proceso de formación ciudadana que haga especial énfasis en la internalización de la norma es algo paradójico teniendo en cuenta que si se pretende formar sujetos autónomos, capaces de conducirse éticamente, llevarlos a aceptar y obedecer una serie de normas diseñadas en la mayoría de los casos por agentes externos, solo fomenta una cultura de la heteronomía.

Y con relación a la definición de unos estándares básicos de competencias que orienten el proceso de formación ciudadana, consideramos que esto es lesivo, en la medida en que se homogenizan aprendizajes como si todos los estudiantes asumieran el mundo de la misma manera. La definición de unos aprendizajes básicos es una postura normalizadora que niega las particularidades de los contextos y contradice en todo sentido el discurso de la libertad y la autonomía, al conducir a las instituciones educativas a desarrollar unos aprendizajes previamente definidos, que tal vez más que satisfacer las necesidades de cono- cimiento buscan la legitimación de discursos que determinen ciertos modos de ser.

\section{UNA PROPUESTA PROVENIENTE DE LA UTOPÍA: “OTRA CIUDADANÍA ES POSIBLE”}

Como resultado de este ejercicio investigativo, evidenciamos que sí es posible desligarse de las disposiciones impuestas por el sistema, revertir el orden establecido, crear maneras diversas de resistencia haciendo uso de nuestra condición de sujetos ético-políticos y, en efecto, hacer que las cosas pasen. Con ello, se pretende demostrar que la micropolítica es la opción que nos queda para trabajar sobre los pequeños espacios en los que habitamos y darle voz a las apuestas que rompen con las dinámicas establecidas para generar un movimiento, una transformación que provenga de la misma inmanencia social, desde abajo (Deleuze y Guattari, 2004).

La apuesta de otra ciudadanía posible puede entenderse como una utopía, que nos sirva para animarnos a caminar. Sumando esfuerzos, a partir de lo encontrado en nuestros metros cuadrados concretos, para construir (ubicados en otras miradas, distintas a las hegemónicas, generalmente eurocéntricas) nuevas maneras de tener que ver con nuestra realidad circundante.

Así, le apostamos a una ciudadanía, que, al resistirse a los embates de la institucionalización del mercado, construye un pensamiento crítico y propio. En la posibilidad de, por ejemplo, tomar partido en contra de condiciones de injusticia e inequidad.

En este orden de ideas, sostenemos con Catherine Walsh, que el desafío es "confrontar desde 'lo propio' y desde lógicas-otras y pensamientos-otros a la deshumanización, el racismo y la racialización, y la negación y destrucción de los campos-otros del saber". Para crear "condiciones radicalmente diferentes de existencia, conocimiento y del poder que podrían contribuir a la fabricación de sociedades distintas" (Walsh, 2005, p. 193).

Para dar este salto, Walsh señala la necesidad de un imaginario-otro de sociedad construido por la interculturalidad. Proyecto que para que sea posible ha de construir "modos otros de poder, saber y ser". 
Enrumbándonos "estratégicamente hacia un pensamiento fronterizo, pero también hacia y por medio de un posicionamiento crítico fronterizo". A través del cual se hagan "visibles otras lógicas y maneras de pensar diferentes a la lógica eurocéntrica y dominante, poniendo a ellas en diálogo y/o disputa con la dominante $y$, en este sentido, poniendo en cuestión su hegemonía como única perspectiva de conocimiento" (Walsh, 2005, pp. 197-198).

Para ello, un camino posible puede ser el de la investigación al margen, entendida "como un posicionamiento y una práctica de producción de conocimiento social, llevado a cabo por sujetos (individuales, colectivos) que proviniendo de las ciencias sociales o no, transgrede la racionalidad disciplinar dominante" (Torres, 2010, p. 192), invitándonos con esto, no solo a contribuir con la construcción de un pensamiento propio, y como ya lo hemos dicho unas líneas antes, distinto al planteado por posiciones hegemónicas y universalizantes del saber, sino, a asumir un pensamiento responsable y comprometido, ética y políticamente, con las transformaciones sociales necesarias para garantizar una vida digna para todos, en los escenarios en donde nos encontremos llevando a cabo nuestra acción investigativa. Para ello, estaremos prestos a asumir como punto de partida en nuestros ejercicios de investigación, la claridad necesaria para acercarnos a las preguntas políticas sobre el por qué, el para qué y para quiénes va a tener sentido la investigación con la que tengamos que ver. Involucrando al máximo en nuestro trabajo investigativo, a las personas pertenecientes al grupo social investigado.

Es más, siguiendo a Alfonso Torres, hemos de anotar que otra ciudadanía puede ser posible si le apostamos a una ética de la justicia de la gratuidad, de la compasión, de la alteridad, de la solidaridad, de lo comunitario incluyente, de la vida, de la hospitalidad, de la contradicción, de la denuncia y de la utopía (Torres, 2009). En últimas a una ética del afecto, del amor.

Otra ciudadanía es posible, si la educación y la pedagogía asumidas con y para los estudiantes con quienes tengamos que ver, de acuerdo con Freire, es liberadora (Freire, 2004), en tanto que su propósito ha de ser el de una amplia concienciación de educandos y educadores, que haga posible la autorreflexión sobre su propio tiempo y espacio. Esto exigirá, por supuesto, rupturas sociales, por lo menos en lo que tiene que ver con nuestros propios metros cuadrados, encaminadas hacia una transformación total de nuestras realidades más cercanas.

Tal educación exige, y creemos que así lo hemos insinuado en nuestra reflexión, una didáctica caracterizada por el afecto, por la participación real de sus sujetos (educadores y estudiantes) y por el diálogo; buscando crear, en educadores y estudiantes, procesos de recreación, de búsqueda, de independencia y, a la vez, de solidaridad (Freire, 2004).

Teniendo en cuenta lo anterior, nuestras prácticas educativas se convierten en punto de reflexión con el fin de encontrar en ellas los aportes pedagógicos y didácticos que las convierten en unas apuestas de resistencia a las imposiciones del sistema educativo enmarcado en una dinámica economicista. Tres estrategias didácticas distintas de formación ciudadana que le apuestan a la micropolítica como una manera de ejercer unas relaciones de poder móviles y flexibles que nos permitan establecer otros modos de ser maestros. Dichas propuestas son las siguientes: "Soy un ser sensible y solidario: otra ciudadanía es posible", desarrollada en el Colegio Camilo Torres Restrepo del municipio de Aguazul, Casanare, donde la afectividad y los espacios libres de condicionamientos son los hilos conductores de los procesos de aprendizaje; "La clase de matemáticas, un ejercicio de participación", en la Escuela Pedagógica Experimental, innovación educativa de Bogotá, donde la participación se da como principio y no como fin; y "El camino del diálogo: camino para la construcción de subjetividades respetuosas de sí mismas y del otro", en la Universidad Distrital Francisco José de Caldas, en Bogotá, donde el diálogo intersubjetivo es el elemento principal para reconocerse a sí mismo y a los otros.

\section{REFLEXIONES FINALES: OTRAS MANERAS DE ASUMIR LA FORMACIÓN CIUDADANA. UNA PROPUESTA ORIENTADA A PARTIR DE UNA PERSPECTIVA DE LA ÉTICA DEL CUIDADO DE SÍ COMO PRÁCTICA DE LIBERTAD}

A partir de lo vislumbrado en el desarrollo de nuestra investigación y en aras de responder de manera concreta al propósito principal de esta, nos permitimos 
presentar algunos principios pedagógicos y didácticos, que podrían orientar una formación ciudadana bajo una perspectiva del cuidado de sí como práctica de libertad.

\section{Principios pedagógicos}

La formación ciudadana, además de proveer a los estudiantes de los conocimientos necesarios para comprender las dinámicas sociopolíticas que caracterizan el sistema democrático que rige al país, debe propender por gestionar en ellos un nuevo ethos caracterizado por el reconocimiento de su condición de sujetos políticos capaces de ejercer unas relaciones de poder (flexibles) sobre sí y sobre los otros; de manera tal que dichas relaciones los lleven a una búsqueda constante de su libertad, entendida como la posibilidad de resistirse a las condiciones impuestas por los códigos socialmente establecidos, para crear sus propios modos de existencia.

Este nuevo ethos debe materializarse en un sujeto crítico y reflexivo frente a su pasado y presente, frente a aquello que lo ha constituido en lo que es, un sujeto sensible y solidario frente a las tragedias de la humanidad, un sujeto propositivo y participativo en la solución de las problemáticas de su contexto, un sujeto capaz de vivir en comunidad en medio del disenso, capaz de moverse entre las imposiciones del sistema represivo, capaz de hacer parte de una consciencia colectiva.

La búsqueda de esa nueva subjetividad para la democracia será el resultado de un proceso formativo desarrollado en ambientes de aprendizaje dispuestos tanto para el trabajo teórico como para el diálogo intersubjetivo, para la crítica, para la reflexión, para la acción, para la participación activa en la construcción de los nuevos aprendizajes y para la afectividad. Ambientes que, aunque inmersos dentro de unas condiciones gubernamentales represivas y homogeneizantes (políticas educativas), les posibiliten a los estudiantes y a los maestros, a través de un ejercicio responsable de poder, identificarlas, reflexionarlas y transformarlas; de tal forma que el resultado de este proceso sea la activación de líneas de fuga que permitan la resistencia, la revolución, el devenir minoritario de cada uno de ellos para no dejarse aprisionar por los códigos socialmente establecidos.
Pensar en constituir una ciudadanía distinta a la imperante equivale, entonces, a pensar en una formación orientada hacia el descubrimiento de nuevas rutas para que el estudiante se autorrealice como sujeto libre; una formación que le permita al estudiante practicar la libertad éticamente, lo que implica tanto el cuidado de sí como de sus semejantes. Con relación al cuidado de sí, la formación ciudadana debe buscar que el estudiante desarrolle la capacidad de ejercer dominio propio, dominio sobre sus deseos, sobre sus fantasmas, de tal forma que no se convierta ni en esclavo de ellos, ni en un tirano.

Con relación al cuidado de los otros, el cuidado de sí implica necesariamente el cuidado de quienes comparten su mismo espacio, pues quien es capaz de gobernarse a sí mismo también es capaz de cuidar de los otros.

En la medida en que el cuidado de sí convierte a quien lo posee en alguien capaz de ocupar en la ciudad, en la comunidad, o en las relaciones interindividuales, el lugar que conviene - ya sea para ejercer una magistratura o para establecer relaciones de amistad. (Foucault, 1994, p. 399).

Aquí, el cuidado puede implicar hacer visibles condiciones que atenten contra la libertad de todos, como gestionar unas relaciones afectivas, cordiales y constructivas con los otros, en aras de crear un ambiente digno para la coexistencia.

En definitiva, el gran principio pedagógico que se propone para una formación ciudadana distinta que transforme tanto la vida de los estudiantes y maestros como a los contextos donde habitan, es una formación orientada hacia la micropolítica planteada por Deleuze y Guattari (2004), donde unos y otros puedan descubrirse como sujetos capaces de transformarse, primero a sí mismos y después a las otros y a las condiciones que los limitan. Una formación ético-política que promueva en los sujetos y en las comunidades una resistencia y una revolución frente a todo lo que restringe su libertad, a partir de un ejercicio ético del poder que les permita trabajar sobre las grietas o espacios donde la dominación no alcanza a permear. Es una apuesta por una transformación de abajo hacia arriba, donde los cambios se dan primero en los propios sujetos, en su entorno inmediato; con 
la esperanza de que luego a partir de esas múltiples acciones individuales se genere un gran movimiento de resistencia colectiva.

Dentro de estos principios pedagógicos, el estudiante asume un rol activo en la constitución de su propia subjetividad a partir del ejercicio crítico y reflexivo como prácticas para el cuidado de sí. A los maestros nos corresponde, en primera instancia, cuidar de nosotros mismos en el sentido de ejercer prácticas de libertad con relación a las imposiciones que limitan nuestro accionar político; imposiciones relacionadas con las disposiciones gubernamentales que lejos de pretender una educación para la autorrealización de los sujetos, buscan subjetivarlos a las dinámicas impuestas por el sistema productivo. Nos corresponde ejercer el rol de guía o consejero que devele a los estudiantes los distintos juegos de verdad que están determinando la existencia de todos para intentar revertirlos; y nos corresponde contagiar o despertar en los estudiantes el deseo de vivir intensamente y con alegría disfrutando cada movimiento, porque amar la vida es amar el cambio; no podemos amar sin desear, sin dejarnos arrastrar por el movimiento mismo de la vida.

\section{Principios didácticos}

Entendiendo como principios didácticos el conjunto de estrategias que los maestros usamos para materializar los procesos de enseñanza, planteamos a continuación algunos que pueden ser significativos para la orientación de la formación ciudadana.

\section{La afectividad como motor para}

la formación ciudadana

No es posible pensar en la idea de formar mejores ciudadanos sin contemplar la dimensión afectiva de los sujetos, teniendo en cuenta que es a partir de los afectos, de las emociones, desde donde se generan las acciones de movimiento o transformación. La vida entera del sujeto se mueve por deseos, por alegrías, por esperanzas.

Una educación para la práctica de la libertad, mediante la cual todo sujeto sea capaz de dignificar su existencia siendo y haciendo lo que desea ser y hacer, será posible solo en la medida en que se contemple que la potencia de todo individuo para transformarse constantemente está en lo que desea, en lo que anhela ser, en el devenir. A partir de los planteamientos de Deleuze, esa potencia es la que posibilita que los sujetos se movilicen constantemente entre un territorio ${ }^{4} \mathrm{y}$ otro (se desterritorialicen), desafiando el orden establecido para renovarse constantemente. Estas variaciones entre un territorio y otro están en función de los encuentros o experiencias que cada sujeto tenga; encuentros más o menos convenientes, que amplían o reducen el territorio, al maximizar o minimizar su potencia; encuentros que afectan a los cuerpos que atraviesan (Lara, 2015).

Así, la formación para una nueva ciudadanía significa proveer a los estudiantes de múltiples experiencias pedagógicas que despierten en ellos su deseo, su amor y goce por la vida, por cada cosa que hacen; que despierten su pasión por existir.

\section{La participación como ágora}

A cambio de la participación basada en la democracia representativa, los diferentes escenarios de la escuela, iniciando con el aula de clase, deben presentarse como un ágora, sitios de conversación y discusión, que permitan la voz directa de los diferentes actores de la comunidad educativa. De esta manera, los estudiantes, los maestros, la parte administrativa y los padres de familia podrían, a través de asambleas, manifestarse y contribuir en los aspectos que consideren pertinentes. Podrían sugerirse espacios de encuentro a partir de necesidades o intenciones particulares.

La apuesta para una formación ciudadana distinta debe surgir en una idea de participación y de democracia en la cual el otro surge como legítimo otro en convivencia con uno; de esta manera, estaríamos reconociendo a todos los miembros de la comunidad educativa, en su heterogeneidad. Legitimar al otro descarta la idea de discriminar y, en consecuencia, términos como inclusión en el aula, no tendrían sentido, pues todos somos seres diversos y particulares.

La reivindicación del diálogo

En esta exposición de principios didácticos, queremos insistir en lo siguiente: a través del diálogo, creemos, se hace posible el favorecimiento de relaciones democráticas y solidarias entre sus actores. Además

4 Entendiendo el territorio como ese otro lugar variable de la acción de la potencia particular de cada persona (Lara, 2015). 
de que con ello se crean condiciones para comprender mejor la realidad que nos circunda, poniendo al servicio del otro el poder de la palabra y del saber. Pues, lo que se expone a través de la palabra, por sobre cualquier otro instrumento de poder, está en la posibilidad de hacerse público, de pertenecer a todos, al conjunto de la población, en medio de la crítica y la controversia.

Es pues, fundamental, en el diálogo, hacer posibles tiempos y espacios para el mutuo respeto por la palabra, el pensamiento y el saber del otro; y para la búsqueda constante del conocimiento y de la creatividad. Pues, como lo hemos insinuado unas líneas antes, en el diálogo no solo las personas y sus vivencias pasan a ser la prioridad por encima de los contenidos y la cantidad de clases, sino que el saber de quienes dialogan se encuentra en la posibilidad de ser reconocido.

Creemos, pues, que en el camino del diálogo, la palabra, la acción y la reflexión van de la mano hacia la transformación del mundo, en la medida en que los sujetos que la pronunciamos, denunciamos con ella la realidad que no nos permite ser y hacer, y anunciamos la realidad en donde podemos ser más (Freire, 2002).

\section{El contexto: la mejor aula de clase}

Enfrentar a los estudiantes a las distintas problemáticas que afectan su entorno inmediato para analizarlas y transformarlas desde sus propias posibilidades es permitirles reconocerse como ciudadanos activos, como sujetos políticos, como actores con poder para transformar esas realidades; les da la posibilidad de sentirse parte de un colectivo social, de fortalecer una identidad y promover una consciencia colectiva. Tomar el contexto como aula de clase significa para estudiantes y maestros reconocer las problemáticas sociales en todas sus dimensiones, interactuar con los diversos actores involucrados, analizar las responsabilidades de cada uno de estos actores, sentirnos parte del problema y plantear alternativas de solución desde nuestra condición de sujetos políticos.

Son espacios donde maestros, estudiantes y demás líderes comunitarios participamos de manera libre y espontánea movidos por unos intereses comunes: participar activamente en el desarrollo social del territorio a través de unas relaciones intersubjetivas marcadas por la sensibilidad, la solidaridad, el compromiso, el respeto y el afecto. Espacios donde cada uno de los actores participantes exploramos nuestras potencialidades y las ponemos a disposición de un objetivo común, entendiendo que nuestra participación es indispensable para transformar positivamente la realidad.

Las estrategias didácticas extracurriculares, particularmente las de carácter comunitario, son la manera más cercana de conducir a los estudiantes hacia un ejercicio consciente de su rol como ciudadano, teniendo en cuenta que además de reconocer y valorar su contexto, les permite participar de su transformación. Son una manera de resistirnos a las condiciones establecidas por el sistema, de desafiar los modelos de aprendizaje tradicionales y de repensar los procesos formativos más allá del aula de clase. Es una manera de entender que el paso por la escuela es una oportunidad para formarse como un sujeto capaz de transformar su vida y su entorno.

\section{REFERENCIAS}

Bustamante, G. (2010). La moda de las "competencias". Recuperado de https://kipdf.com/la-moda-de-las-competencias_5ae5146e7f8b9acd258b45cd. html

Carr, W., y Kemmis, S. (1988). La investigación-acción como ciencia educativa crítica. En Teoría critica de la enseñanza. La investigación acción en la formación del profesorado (pp. 190-223). Barcelona: Editorial Martínez Roca

Chaux, E., Lleras, J. y Velásquez, A. M. (2004). Competencias ciudadanas: de los estándares al aula. Una propuesta de integración a las áreas académicas. Bogotá: Uniandes.

Comins, M. I. (2009). La ética del cuidado y las tareas de atención y cuidado. En Filosofía del cuidar. Una propuesta coeducativa para la paz (pp. 87-142). Barcelona: Icaria Editorial S.A.

Deleuze, G., y Guattari, F. (2004). Mil mesetas capitalismo y esquizofrenia. Valencia: Pre-Textos.

Fomet-Betancourt, R., Becker, H. y Gómez-Muller, A. (1984). La ética del cuidado de uno mismo como práctica de la libertad. Concordia, 6, 99-116.

Foucault, M. (1994). Estética, ética y herméneutica. Obras esenciales. Vol. III. (Trad. Á. Gabilondo). París: Paidós.

Foucault, M. (2007). Nacimiento de la Biopoítica. Curso en el colegio de Francia 1978-1979. Buenos Aires: Fondo de Cultura Económica. 
Freire, P. (2002). Pedagogía del oprimido (Trad. J. Mellano). Madrid, Siglo XXI.

Freire, P. (2004). La educación como práctica de la libertad (Trad. L. Ronzoni). Madrid, Siglo XXI.

Lara, L. (2015). Teorías afectivas vintage. Apuntes sobre Deleuze, Bergson y Whitehead. Cinta de Moebio. Revista de Epistemología de Ciencias Sociales, 17-36. Recuperado de http://www.facso.uchile.cl/publicaciones/ moebio/info.html.

Maturana, H. (1994). La democracia es una obra de arte. Bogotá: Editorial Magisterio.

Ministerio de Educación Nacional (MEN). (2011). Orientaciones para la institucionalización de las competencias ciudadanas . Cartilla 1. Brújula, Programa de competencias ciudadanas. Bogotá.
Nussbaum, M. (2010). Por qué la democracia necesita de las humanidades. Educar ciudadanos: los sentimientos morales (y antimorales). En Sin fines de lucro (pp. 51-74). Buenos Aires: Kats Editores.

Ruiz Silva, A. R., y Chaux Torres, E. (2005). La formacion de competencias ciudadanas. Bogotá: Ascofade.

Torres, A. (2009). Educación popular y paradigmas emancipadores. La piragua (30), 11-32.

Torres, A. (2010). Pensar la investigación desde el margen de las ciencias sociales. En H. Valencia, ¿Cómo pensar las ciencias sociales hoy? (pp. 187-205). Bogotá: Universidad Pedagógica Nacional.

Walsh, C. (2005). Pensamiento crítico y matriz (De) colonial. Reflexiones latinoamericanas. Quito: Universidad Andina Símon Bolivar. 